\title{
A Big Bang-Big Crunch Optimization Based Approach for Interval Type-2 Fuzzy PID Controller Design
}

\author{
Tufan Kumbasar \\ Control Engineering Department \\ Istanbul Technical University \\ Istanbul, Turkey \\ kumbasart@itu.edu.tr
}

\author{
Hani Hagras \\ The Computational Intelligence Centre \\ University of Essex \\ Colchester, United Kingdom \\ hani@essex.ac.uk
}

\begin{abstract}
In this paper, we will present a big bang-big crunch optimization (BB-BC) based approach for the design of an interval type-2 fuzzy PID controller. The implemented global optimization algorithm has a low computational cost and a high convergence speed. As a consequence, the BB-BC method is a very efficient search algorithm when the number of the optimization parameters is relatively big. The optimized type-2 fuzzy controller is compared with PID and type-1 fuzzy PID controllers which were optimized with either the BB-BC optimization method or conventional design strategies. The paper will also show the effect the extra degrees of freedom provided by the antecedent interval type-2 fuzzy sets on the closed loop system performance. We will present a comparative study performed on the highly nonlinear cascaded tank process to show the superiority of the optimized interval type-2 fuzzy PID controller compared to its optimized PID, type-1 counterparts.
\end{abstract}

Keywords-interval type-2 fuzzy sets; interval type-2 fuzzy PID; nonlinear control; Big Bang-Big Crunch Optimization.

\section{INTRODUCTION}

In the recent years, Interval Type-2 Fuzzy Logic Controllers (IT2-FLC) have been widely used to control nonlinear systems where the IT2-FLCs demonstrated significant performance improvements. The internal structure of the IT2-FLC is similar to the type-1 counterpart. However, the major differences are that IT2-FLCs employ interval type-2 fuzzy sets (rather than type-1 fuzzy sets) and the IT2-FLCs process Interval Type-2 Fuzzy Sets (IT2-FSs) and thus the IT2FLC has the extra type-reduction process [1], [2]. It has been shown in various works that IT2-FLCs achieve better control performance because of the additional degree of freedom provided by the Footprint of Uncertainty (FOU) in their Membership Functions (MFs) [3], [4]. Consequently, IT2FLCs have attracted much research interest, especially in control applications [3-9]. In [10], [11], it has been reported that the IT2-FLCs are generally more robust than type-1 counterparts. However, IT2-FLCs are difficult to analyze and design since their internal structure is typically more complex than their type-1 counterpart [12]. In this context, several studies have been performed in order to design the IT2-FLCs [13-15]. Nevertheless, a systematic design for type-2 fuzzy controllers is still a challenging problem due to the main difficulty in determining the IT2-FSs and rulebase. Recently, several studies have employed evolutionary algorithms for the design of IT2-FLCs $[5,15]$.

In this paper, we will present the novel application of the evolutionary algorithm called Big Bang- Big Crunch (BB-BC) optimization [16] to optimize the antecedent parameters of the Interval Type-2 Fuzzy PID (IT2-FPID) controller for a highly nonlinear cascaded two tank process. Since the number of the design parameters of the IT2-FPID is relatively big, the BB-BC global optimization algorithm has been preferred as it has a low computational cost and a high convergence speed. The performance of the BB-BC Optimized IT2-FPID (OIT2-FPID) controller will be compared with a BB-BC Optimized Type-1 Fuzzy PID (OT1-FPID) and PID (OPID) controllers. In order to make a fair comparison, the rule base and scaling factors are kept fixed for the fuzzy controller structures while only the antecedent fuzzy sets parameters are optimized for certain reference trajectory. It will be illustrated that the extra degree of freedom of the antecedent IT2-FSs gives the BB-BC method an opportunity to find a more optimal solution than those obtained for the optimized OPID and OT1-FPID with respect to the Integral of the Absolute Error (IAE) performance index. The optimized controllers are also compared with an Internal Model Control based PID (IMC-PID) and an Internal Model Control based Type-1 Fuzzy PID (IMC-T1-FPID) controller structures. A detailed comparative study has been conducted to show that the control performance of the OIT2-FPID is better in different operating points even at those at which the controllers are not optimized or tuned and is more robust against noise and disturbances when compared to its optimized T1-FPID and traditional PID counterparts.

Section II will present the BB-BC optimization algorithm. Section III will briefly describe the nonlinear two cascaded tank process. Section IV will present the control design strategies for the conventional PID, T1-FPID and IT2-FPID structures. Section V will present the simulation studies and results while Section VI will present the conclusions and future work.

\section{A BRIEF OVERVIEW ON BIG BANG-BIG CRUNCH OPTIMIZATION}

In [16], a new evolutionary algorithm named Big Bang Big Crunch (BB-BC) was proposed. The leading advantage of BB- 
$\mathrm{BC}$ is the high convergence speed and the low computation time. The working principle of this method can be explained as the transformation of a convergent solution to a chaotic state and then back to a single tentative solution point. The BB-BC algorithm has been implemented in various engineering applications where the computational time and convergence time are important factors. The efficiency of this evolutionary algorithm has been demonstrated especially where the optimization problem must be solved in relatively small sampling times such as in inverse fuzzy model control and fuzzy model adaptation [17]. Moreover, in the optimization of highly nonlinear engineering problems such as controller design [18], design of space trusses [19], and size reduction of space trusses [20], this algorithm has been preferred because of its high convergence speed.

The Big Bang-Big Crunch (BB-BC) optimization method proposed in [16] consists of two main steps. The first step is the "Big Bang" phase where candidate solutions are randomly distributed over the search space and the next step is the "Big Crunch" phase where a contraction procedure calculates the center of mass for the population. The initial Big Bang population is randomly generated over the entire search space just like the other evolutionary search algorithms. All subsequent "Big Bang" phases are randomly distributed about the center of mass or the best fit individual in a similar fashion [16]. After the "Big Bang" phase, a contraction procedure is applied as the "Big Crunch" phase to form a center or a representative point for further "Big Bang" operations. In this phase, the contraction operator takes the current positions of each candidate solution in the population and its associated cost function value and computes a center of mass. The center of mass can be computed as:

$$
x_{c}=\frac{\sum_{i=1}^{N} \frac{1}{f^{i}} x_{i}}{\sum_{i=1}^{N} \frac{1}{f^{i}}}
$$

where $x_{c}$ is the position of the center of mass, $x_{i}$ is the position of the candidate, $f$ is the cost function value of the $i^{\text {th }}$ candidate, and $N$ is the population size. Instead of the center of mass, the best fit individual can also be chosen as the starting point in the "Big Bang" phase. The new generation for the next iteration "Big Bang" phase is normally distributed around $x_{c}$. The new candidates around the center of mass are calculated by adding or subtracting a normal random number whose value decreases as the iterations elapse. This can be formalized as

$$
x^{\text {new }}=x_{c}+\frac{r \alpha\left(x_{\max }-x_{\min }\right)}{k}
$$

where $r$ is random number; $\alpha$ is a parameter limiting the size of the search space, $x_{\max }$ and $x_{\min }$ are the upper and lower limits; and $k$ is the iteration step. In [16] the working principle of this evolutionary method is explained as to transform a convergent solution to a chaotic state which is a new set of solutions.

\section{ThE Two CASCADED TANK Process}

The schematic diagram of the two-cascaded tank process is illustrated in Fig. 1. The first tank ("Tank 2") is a straight tank with a cross sectional area $A_{2}$. The second tank ("Tank 1") is an inclined tank. The cross-sectional area is related to the level of the tank. The angle of its sidewall is $\beta$, and the width of Tank 1 is $L$.

The differential equation related to the tank level system is given as:

$$
\begin{aligned}
& \frac{d h_{1}}{d t}=\frac{-\gamma_{1} \sqrt{2 g h_{1}}+\gamma_{2} \sqrt{2 g h_{2}}}{2\left(d+h_{1} \tan \beta\right) L} \\
& \frac{d h_{2}}{d t}=-\frac{\gamma_{2}}{A_{2}} \sqrt{2 g h_{2}}+\frac{1}{A_{2}} F_{i n}(t)
\end{aligned}
$$

The system output is defined as:

$$
h=\left[\begin{array}{ll}
1 & 0
\end{array}\right]\left[\begin{array}{l}
h_{1} \\
h_{2}
\end{array}\right]
$$

The process parameters are: $A_{2}=85 \mathrm{~cm} 2, \gamma_{l}=0.3539 \mathrm{~cm}^{2}$, $\gamma_{2}=0.3027 \mathrm{~cm} 2, L=20 \mathrm{~cm}, \beta=5.1^{\circ}, d=3.32 \mathrm{~cm}$, the acceleration of gravity $g=9.8 \mathrm{~ms}^{-2}$ and the initial levels are $h_{10}=5 \mathrm{~cm}, h_{20}=$ $5 \mathrm{~cm}$ while the inlet flow range is $0-100 \mathrm{~cm}^{3} / \mathrm{s}$ [21].

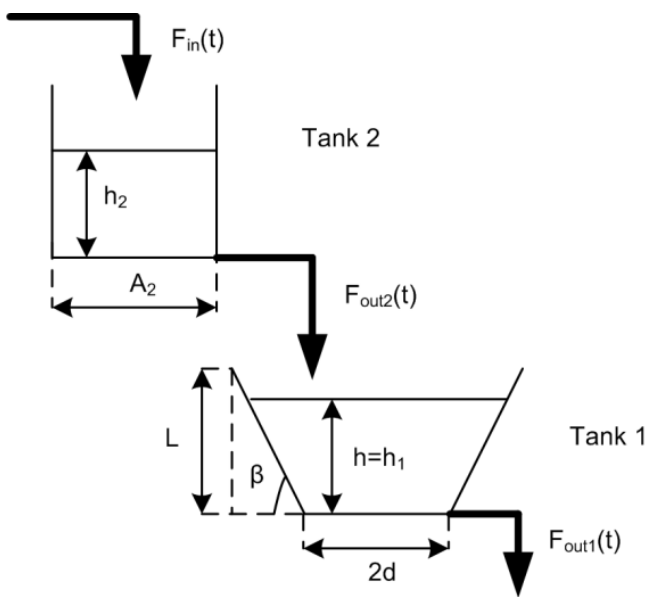

Fig. 1. Illustration of the two-cascaded tank process

\section{The Controllers Design StRATEGIES}

In this section, we will present the design strategies of the implemented fuzzy and conventional PID controllers. We will start by presenting the two design strategies employed for the conventional PID controller structure. We will then present the two design strategies employed for T1-FPID. Finally, we will present the design strategy employed for the design of the IT2FPID controller.

\section{A. Conventional PID Controller Design Methods}

In this subsection, we will present the employed design strategies for the conventional PID controllers. The first design strategy employs an Internal Model Control based PID controller based on the First Order Plus-Time Delay (FOPDT) model approximation [22]. As it is well-known, high order or nonlinear processes can be represented by FOPDT models that correspond to [23]:

$$
G(s)=\frac{K e^{-L s}}{T s+1}
$$




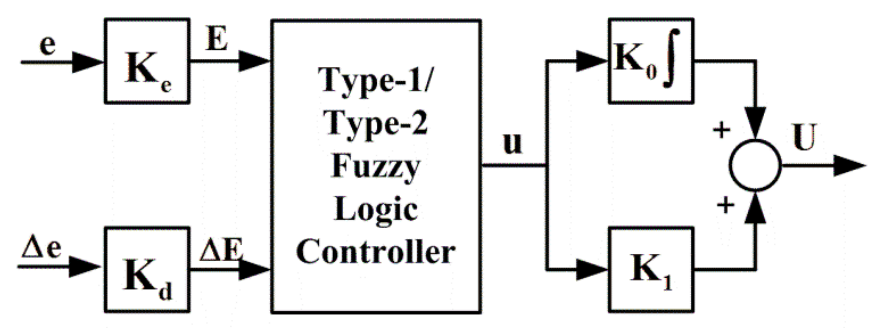

(a)

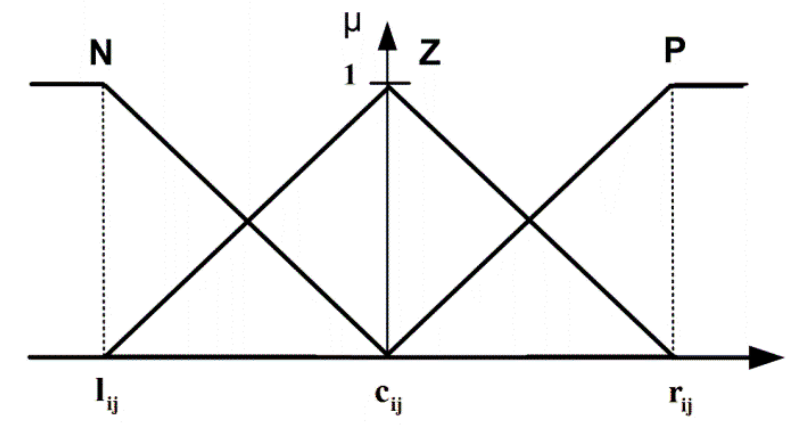

(c)

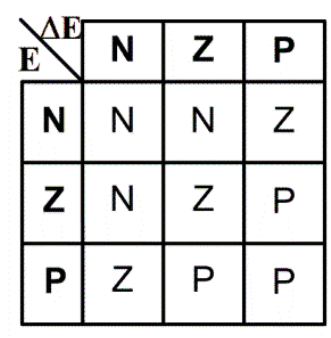

(b)

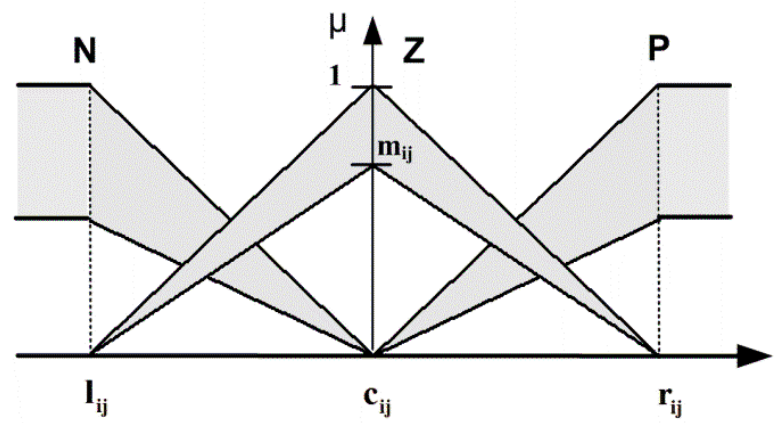

(d)

Fig. 2. (a) T1-FPID/ IT2-FPID controller structure (b) Rule base (c) Type-1 Fuzzy Membership Functions (d) Interval Type-2 Fuzzy Membership Functions

When the nonlinear tank system is modeled as FOPDT for the operating point $\left(h_{I}=10 \mathrm{~cm}, F_{i n}=50 \mathrm{~cm}^{3} / \mathrm{s}\right)$, the model parameters are obtained based on the step response method as follows [23]: $K=0.221, T=50 \mathrm{~s}, L=10 \mathrm{~s}$.

In the IMC based design method [22], the PID structure inherits a low pass filter and is defined as follows:

$$
I M C-P I D(s)=K_{c}\left(1+\frac{1}{\tau_{I} s}+\tau_{D} s\right)\left(\frac{1}{1+\tau_{f} s}\right)
$$

Where $K_{c}$ is the proportional gain $\tau_{I}$ is the integral gain, $\tau_{D}$ is the derivative gain and $\tau_{f}$ is the time constant of filter. These parameters are defined as:

$$
\begin{aligned}
& K_{c}=\frac{T+0.5 L}{K\left(\tau_{c}+L\right)} ; \tau_{I}=T+0.5 L ; \\
& \tau_{D}=\frac{0.5 L \tau}{T+0.5 L} ; \tau_{f}=\frac{0.5 L \tau_{c}}{\tau_{c}+L}
\end{aligned}
$$

In this design procedure, the desired closed-loop time constant $\tau_{c}$ is the only design parameter [22]. For the two cascaded tank system, the time constant $\tau_{c}$ is chosen as 20 seconds so as to obtain moderate closed loop system response with a low IAE value for the operating point. Then, the controller parameters are calculated as $K_{c}=8.29, \tau_{I}=49.5, \tau_{\mathrm{D}}=6.06$ and $\tau_{f}=4$.

In the second PID design strategy, the three parameters $\left(K_{c}, \tau_{I}, \tau_{D}\right)$ of a conventional PID controller are optimized so as to minimize the IAE performance index via the BB-BC optimization algorithm. The IAE is defined as:

$$
I A E=\int_{0}^{\infty}|r(t)-y(t)| d t
$$

where $y(t)$ is the process output and $r(t)$ is the reference value.

The transfer function of the optimized PID controller is given as:

$$
\operatorname{OPID}(s)=K_{c}\left(1+\frac{1}{\tau_{I} s}+\tau_{D} s\right)
$$

\section{B. The Type-1 and Interval Type-2 Fuzzy PID Controller Design Methods}

In this subsection, the design strategies for the implemented type- 1 and the interval type-2 fuzzy PID controllers are presented. Here, the standard two input fuzzy PID controllers as illustrated in Fig.2a is preferred and used [24]. The input to the fuzzy PID controllers are the error $(e=r-y)$ and the change of error $(\Delta e)$, the output of the FLCs is the change of the control signal $(u)$. The input scaling factors $K_{e}$ for error and $K_{d}$ for the change of error normalize the inputs to the range in which the membership functions of the inputs are defined. While $K_{0}$ and $K_{l}$ are the output scaling factors.

In the implemented T1-FLCs and IT2-FLC structures, a symmetrical $3 \times 3$ rule base is used as shown in Fig. $2 b$. Here, the outputs are defined with three crisp singleton consequents (Negative $(N)=-1, \quad$ Zero $(Z)=0$, Positive $(P)=0)$. The implemented T1-FLCs use the product implication and the centroid defuzzification method while the IT2-FLC uses the center of sets type reduction/ defuzzification method [2].

For the sake of simplicity, the inputs of all FLC structures are defined with three $(J=3)$ triangular type membership functions and are denoted as $N$ (Negative), $Z$ (Zero) and $P$ (Positive). The type-1 fuzzy membership function of the T1FLC is defined with three parameters $\left(l_{i j}, c_{i j}, r_{i j} ; i=1,2, j=1,2,3\right)$, as shown in Fig.2c, while the interval type-2 fuzzy membership 
function is defined with four parameters $\left(l_{i j}, c_{i j}, r_{i j}, m_{i j} ; i=1,2\right.$, $\mathrm{j}=1,2,3$ ), as shown in Fig. $2 \mathrm{~d}$.

In the first T1-FPID design strategy, an IMC based tuning method is used to determine the scaling factors for a type-1 fuzzy PID based on the FOPDT model approximation [25]. In this fuzzy structure, the antecedent triangular membership functions must strictly partition the input domain, i.e., $50 \%$ overlapping. The parameters of the antecedent membership functions of the IMC-T1-FPID are tabulated in Table I. In this case, the input and output scaling factors can then be calculated via the following equations:

$$
K_{0}=\frac{1}{K K_{e}\left(\tau_{c}+\frac{L}{2}\right)} ; K_{1}=\beta K_{0} ; K_{d}=\alpha K_{e}
$$

where

$$
\begin{aligned}
& \beta=\max (T, L / 2), \alpha=\min (T, L / 2) \\
& K_{e}=\frac{1}{r\left(t_{f}\right)-y\left(t_{f}\right)}
\end{aligned}
$$

where $r\left(t_{f}\right)$ and $y\left(t_{f}\right)$ are the values of the reference and system output at the time of the reference variation $\left(t=t_{f}\right)$, respectively. Then, the scaling factors are calculated as:

$$
K_{d}=5 K_{e}, K_{0}=5.525, K_{I}=276.25
$$

In the second fuzzy PID design strategy, the parameters of the antecedent membership functions are optimized via the BB$\mathrm{BC}$ method such that to minimize the IAE performance index given in (8) for both the type-1 and interval type-2 fuzzy PID controllers. In the optimization of the fuzzy PID controllers, the $\mathrm{BB}-\mathrm{BC}$ will only tune the parameters of the antecedent MFs, while the scaling factors, given in (12), and the rule base, given in Fig.2b, are kept fixed.

The antecedent membership functions of OT1-FPID controller that are labeled as the " $N$ " and " $P$ " are defined for each input with two parameters each which are $\left(c_{i l}, r_{i l}\right)$ and $\left(l_{i 3}, c_{i 3}\right.$, $i=1,2)$, respectively while the one labeled as ' $Z$ ' is defined with three parameters $\left(l_{i 2}, c_{i 2}, r_{i 2}, \mathrm{i}=1,2\right)$. Hence for two inputs, the total number of the parameters to be optimized for the OT1FPID design is then $2 \times 7=14$. Whereas, the antecedent IT2-FSs of OT1-FPID controller that are labeled as the " $N$ " and " $P$ " for each input are defined with three parameters each which are $\left(c_{i l}, r_{i l}, m_{i l}\right)$ and $\left(l_{i 3}, c_{i 3}, m_{i 3}, i=1,2\right)$, respectively while the IT2FS labeled as " $Z$ ' is defined with four parameters $\left(l_{i 2}, c_{i 2}, r_{i 2}\right.$, $\left.m_{i 2}, i=1,2\right)$. Consequently, for the two inputs the total numbers to be optimized for the OIT2-FPID design is $2 \times 10=20$. It is obvious that, the OIT2-FPID has 6 more parameters, i.e. extra degrees of freedom.

\section{IAE performance evaluation of the optimized controller structures}

In this subsection, the optimization results of the OPID, OT1-FPID and OIT2-FPID controller structures for the cascaded nonlinear tank system are examined and compared. Since the aim of this subsection is to make a fair comparison, the population size and the number of iterations for all three $\mathrm{BB}-\mathrm{BC}$ based design strategies have been chosen as 100 and 1000 , respectively. In the optimization studies, since the nonlinearity is related to the level of the both tanks, a reference trajectory with the values of 12,20 and $29 \mathrm{~cm}$, in successive order, have been applied to all three controller structures. The design parameters of the controllers are optimized such that to minimize total IAE performance value for the reference trajectory. For the desired reference trajectory, the best values of the OPID controller parameters are found as $K_{c}=23.96$, $\tau_{I}=49.92, \tau_{D}=7.2$. The best obtained parameter set for the OT1-FPID and OIT2-FPID controllers are tabulated in Table I. The obtained best IAE values of the OPID, OT1-FPID and OIT2-FPID are 1677.0868, 1109.5860 and 952.4753 respectively.In Fig.3, the variations of the IAE values are illustrated. It can be clearly seen that the BB-BC optimizing the OPID and OT1-FPID converge with a much higher IAE values than the OIT2-FPID which ends up with a lower IAE value. This shows that the extra degrees of freedom enable the BB$\mathrm{BC}$ to find a more optimal solution than those obtained by OPID and OT1-FPID for the desired reference trajectory with the values of 12,20 and $29 \mathrm{~cm}$.

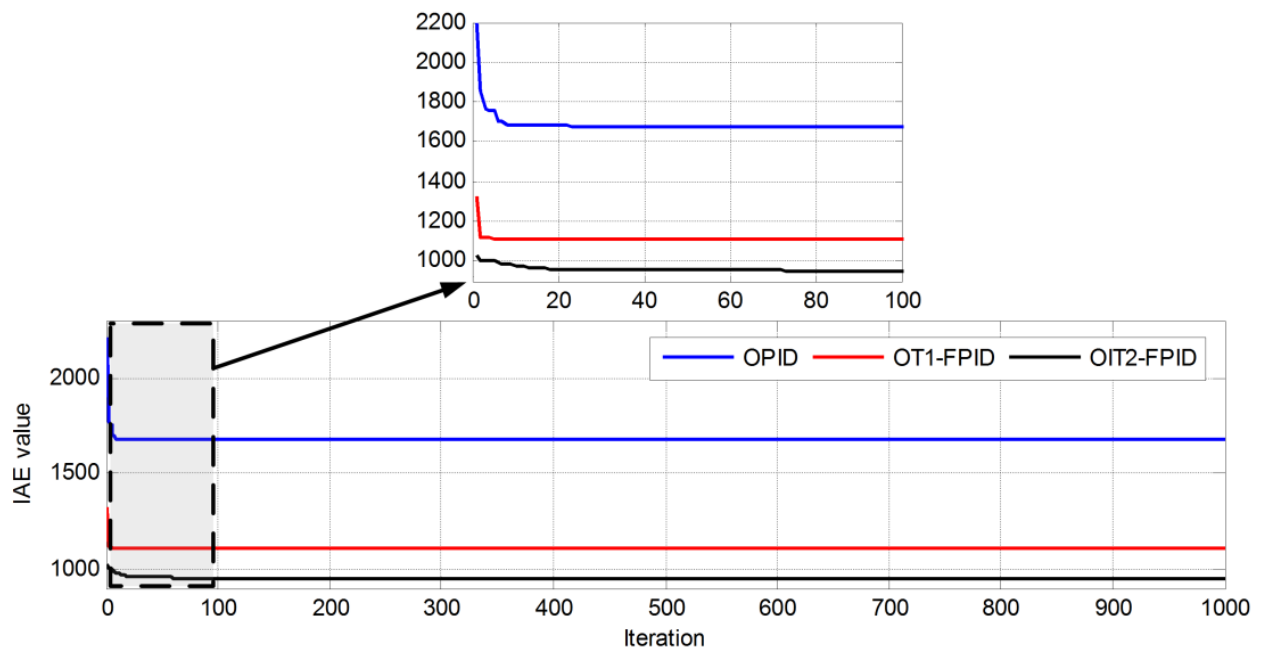

Fig. 3. The variations of the IAE values 
TABLE I. PARAMETERS OF THE ANTECEDENT MFs

\begin{tabular}{|c|c|c|c|c|c|c|}
\hline \multirow{7}{*}{ 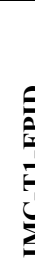 } & & & \multicolumn{4}{|c|}{ Parameters of the MFs } \\
\hline & & & $l$ & $c$ & $r$ & $m$ \\
\hline & & $N$ & & -1.0000 & 0.0000 & \\
\hline & $E$ & $\boldsymbol{Z}$ & -1.0000 & 0.0000 & 1.0000 & \\
\hline & & $\boldsymbol{P}$ & 0.0000 & 1.0000 & & \\
\hline & & $N$ & & -1.0000 & 0.0000 & \\
\hline & $\Delta \boldsymbol{E}$ & $\boldsymbol{Z}$ & -1.0000 & 0.0000 & 1.0000 & \\
\hline 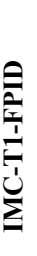 & & $\boldsymbol{P}$ & 0.0000 & 1.0000 & & \\
\hline \multirow{6}{*}{$\frac{1}{2}$} & & $N$ & & -0.5404 & 0.0048 & \\
\hline & $E$ & $\boldsymbol{Z}$ & -0.5134 & 0.0484 & 1.4999 & \\
\hline & & $\boldsymbol{P}$ & 0.0020 & 1.4928 & & \\
\hline & & $N$ & & -0.5244 & 0.0157 & \\
\hline & $\Delta E$ & $\boldsymbol{Z}$ & -0.5162 & 0.0017 & 1.5000 & \\
\hline & & $\boldsymbol{P}$ & 0.0104 & 1.5002 & & \\
\hline \multirow{6}{*}{ 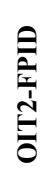 } & & $N$ & & -0.5005 & 0.0000 & 0.7287 \\
\hline & $E$ & $\boldsymbol{Z}$ & -0.5317 & 0.0131 & 1.8368 & 0.2484 \\
\hline & & $\boldsymbol{P}$ & 0.0975 & 1.8632 & & 0.4676 \\
\hline & & $N$ & & -0.5792 & -0.0623 & 0.1818 \\
\hline & $\Delta E$ & $\boldsymbol{Z}$ & -0.3813 & 0.0013 & 0.9095 & 0.1167 \\
\hline & & $\boldsymbol{P}$ & 0.0356 & 0.9487 & & 0.1949 \\
\hline
\end{tabular}

Since the BB-BC optimization method is a stochastic global search method, a statistical evaluation is performed. The optimization routine for each of the controller structures is repeated 5 times and the best IAE value for each run are recorded. In Table II, the best, the worst and the average IAE values for the five runs are tabulated. Although IT2-FPID has the largest number of parameters to be optimized, it has a lower IAE value in all three cases.

TABLE II. Best, Average and Worst IAE Values For The Five TRIALS

\begin{tabular}{cccc}
\hline & \multicolumn{3}{c}{ IAE performance } \\
OPID & Best & Average & Worst \\
\cline { 2 - 4 } OT1-FPID & 1677.0868 & 1731.2086 & 1759.7616 \\
OIT2-FPID & 0952.4756 & 1148.8646 & 1240.7341 \\
\hline
\end{tabular}

\section{Closed Loop Control Performance Analyses}

In this section, different analyses are presented to investigate the closed loop control performances of the IMC-
PID, OPID, IMC-T1-FPID, OT1-FPID and OIT2-FPID. In order to make a fair comparison, three performance measures are considered. Two of these performance measures are selected from the classical transient system response criteria; namely, the settling time $(T s)$, and the overshoot (OS\%) while the other performance measure is the IAE.

At first, we will analyze the control performances for the reference trajectory at which the controllers are designed. Next, the ability of the controllers to handle with output noise is investigated for the same reference trajectory. Moreover, the ability of the controllers to cope with nonlinear dynamics is investigated by defining a reference trajectory at which the controllers are neither optimized nor tuned. Finally, the input and output disturbance rejection performances of the implemented controllers are presented and compared.

\section{A. Trajectory Control Performance Analyses}

In the first performance study, the reference trajectory is examined at which the controllers are optimized. The control signals and process outputs of the implemented controller structures are illustrated in Fig.4. As it can be clearly seen in Table III, the OIT2-FPID structure improves the overall performance of the process much better in every sense compared to the other controllers. For instance, for the reference value variation from $20 \mathrm{~cm}$ to $29 \mathrm{~cm}$ (without output noise), the OT1-FPID has the performance measures $\mathrm{Ts}=222 \mathrm{~s}$, $\mathrm{OS}=24 \%$ and a total IAE value of 1109.5860 as tabulated in Table III. For this operating point, the OIT2-FPID reduces the overshoot to $7 \%$; it also decreases the settling time to about $150 \mathrm{~s}$ and the total IAE value to 952.4753 when it is compared to the OT1-FPID. Similar comments can be made for the other two reference variations.

Moreover, in order to understand how much the controllers are robust against noise, white noise is added at the output of the process. The simulation is then repeated for the same reference trajectory. The process outputs are illustrated in Fig.5. As it can be clearly seen, the OIT2- PID is more robust against noise compared to the fuzzy and conventional structures. The performance measures are tabulated in in Table III.

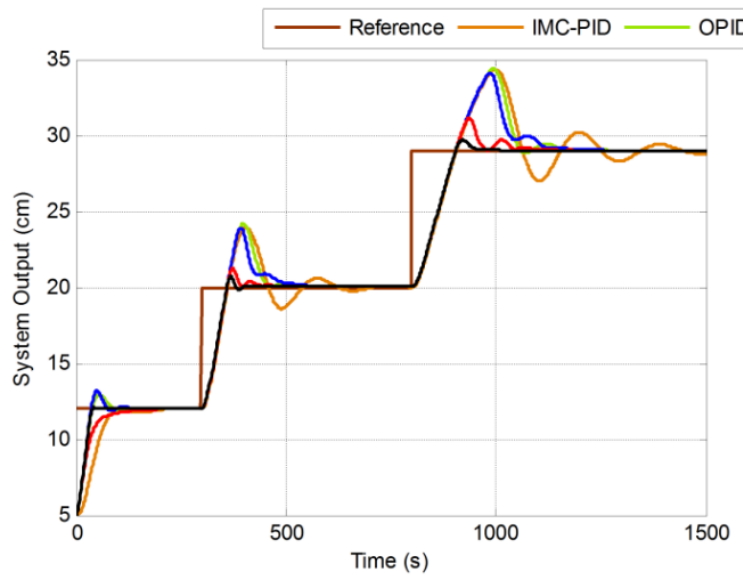

(a)

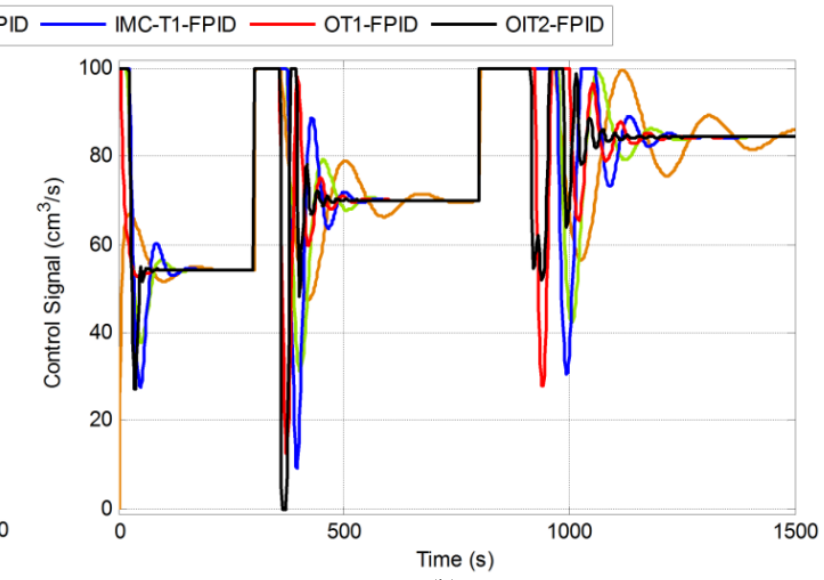

(b)

Fig. 4. Illustration of the (a) process outputs and (b) control signals for first trajectory control performance without output noise. 
TABLE III. Performance COMPARISONS For the First TrajeCtory CONTROL PERFormance Study

\begin{tabular}{|c|c|c|c|c|c|c|c|c|}
\hline & & \multicolumn{6}{|c|}{ TRANSIENT PERFORMANCE } & \multirow{3}{*}{ IAE } \\
\hline & & \multicolumn{2}{|c|}{$5-12 \mathrm{~cm}$} & \multicolumn{2}{|c|}{$12-20 \mathrm{~cm}$} & \multicolumn{2}{|c|}{$20-29 \mathrm{~cm}$} & \\
\hline & & Ts & OS & Ts & OS & Ts & OS & \\
\hline \multirow{2}{*}{ IMC-PID } & w/o Noise & $90 \mathrm{~s}$ & $00 \%$ & $296 \mathrm{~s}$ & $48 \%$ & $432 \mathrm{~s}$ & $58 \%$ & 2265.4269 \\
\hline & w/ Noise & $92 \mathrm{~s}$ & $00 \%$ & $310 \mathrm{~s}$ & $50 \%$ & $612 \mathrm{~s}$ & $65 \%$ & 2343.8366 \\
\hline \multirow{2}{*}{ OPID } & w/o Noise & $80 \mathrm{~s}$ & $23 \%$ & $146 \mathrm{~s}$ & $52 \%$ & $248 s$ & $58 \%$ & 1677.0868 \\
\hline & w/ Noise & $88 \mathrm{~s}$ & $23 \%$ & $160 \mathrm{~s}$ & $63 \%$ & $436 \mathrm{~s}$ & $65 \%$ & 1851.3422 \\
\hline \multirow{2}{*}{ IMC-T1-FPID } & w/o Noise & $68 \mathrm{~s}$ & $18 \%$ & $174 \mathrm{~s}$ & $45 \%$ & $300 \mathrm{~s}$ & $57 \%$ & 1630.9592 \\
\hline & w/ Noise & $120 \mathrm{~s}$ & $48 \%$ & $186 \mathrm{~s}$ & $55 \%$ & $330 \mathrm{~s}$ & $64 \%$ & 1854.6612 \\
\hline \multirow{2}{*}{ OT1-FPID } & w/o Noise & $90 \mathrm{~s}$ & $00 \%$ & $086 \mathrm{~s}$ & $15 \%$ & $222 \mathrm{~s}$ & $24 \%$ & 1109.5860 \\
\hline & w/ Noise & $102 \mathrm{~s}$ & $45 \%$ & $226 \mathrm{~s}$ & $25 \%$ & $422 \mathrm{~s}$ & $38 \%$ & 1906.9655 \\
\hline \multirow{2}{*}{ OIT2-FPID } & w/o Noise & $36 \mathrm{~s}$ & $00 \%$ & $074 \mathrm{~s}$ & $07 \%$ & $130 \mathrm{~s}$ & $07 \%$ & 0952.4753 \\
\hline & w/ Noise & $74 \mathrm{~s}$ & $22 \%$ & $110 \mathrm{~s}$ & $11 \%$ & $150 \mathrm{~s}$ & $01 \%$ & 1342.5881 \\
\hline
\end{tabular}

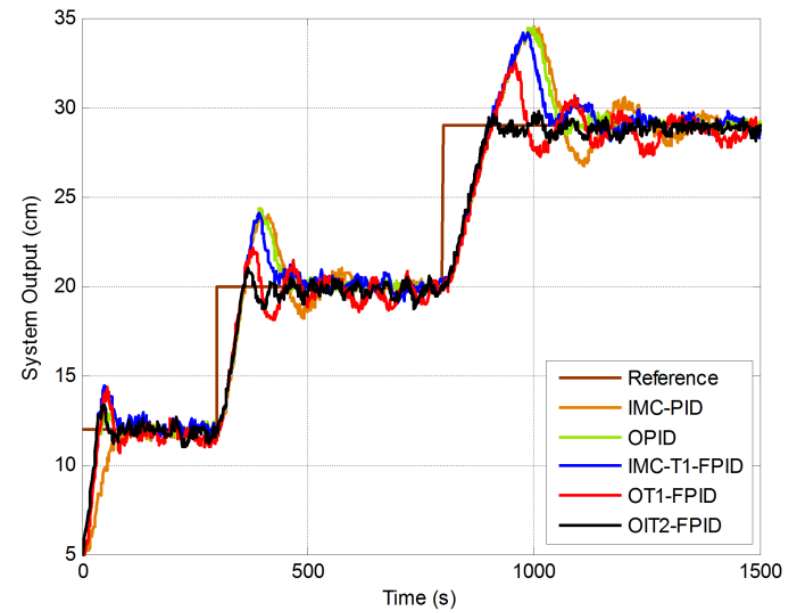

Fig. 5. Illustration of the process outputs for the first trajectory control performance with output noise.

The performances of the designed controllers have been also tested for another reference trajectory. Since the nonlinearity is related to the level of the both tanks, the control performances of the implemented controllers is investigated for two operating points at which they are not optimized or tuned. Thus, a reference trajectory with the values of 26 and $16 \mathrm{~cm}$, in successive order, has been chosen in order to examine how the controllers handle nonlinearity. As it has been shown in Fig.6 that the OIT2-FPID structure provides better transient performances than the type-1 fuzzy and conventional controller structures for this varying reference values. For instance, for the reference variation from $26 \mathrm{~cm}$ to $16 \mathrm{~cm}$, the OIT2-FPID produces superior control performance than the other controllers as its process response has the smallest overshoot and settling time. The performance measures are tabulated in in Table IV. It can be concluded that, the OIT2- FPID structure has the ability to cope better with the nonlinear dynamics compared to the other controllers.

TABLE IV. PERFormance COMPARISONS For THE SECOND TRAJECTORY CONTROL PERFORMANCE STUDY

\begin{tabular}{|c|c|c|c|c|c|}
\hline & \multicolumn{4}{|c|}{ TRANSIENT PERFORMANCE } & \multirow{2}{*}{ IAE } \\
\hline & \multicolumn{2}{|c|}{$5-26 \mathrm{~cm}$} & \multicolumn{2}{|c|}{$26-16 \mathrm{~cm}$} & \\
\hline & Ts & OS & Ts & OS & \\
\hline IMC-PID & $456 \mathrm{~s}$ & $34 \%$ & $190 \mathrm{~s}$ & $42 \%$ & 33.7015 \\
\hline OPID & $306 \mathrm{~s}$ & $41 \%$ & $148 \mathrm{~s}$ & $40 \%$ & 34.6948 \\
\hline IMC-T1-FPID & $358 \mathrm{~s}$ & $33 \%$ & $160 \mathrm{~s}$ & $40 \%$ & 33.7506 \\
\hline OT1-FPID & $210 \mathrm{~s}$ & $14 \%$ & $216 \mathrm{~s}$ & $73 \%$ & 28.9817 \\
\hline OIT2-FPID & $162 \mathrm{~s}$ & $09 \%$ & $118 \mathrm{~s}$ & $28 \%$ & 27.9225 \\
\hline
\end{tabular}

\section{B. Disturbance Rejection Performance Analyses}

This subsection examines the input and output disturbance rejection performances of the implemented controllers. In this context, it is assumed that the process is steady state at the operating point $\left(h_{l}=20 \mathrm{~cm}, F_{i n}=70 \mathrm{~cm}^{3} / \mathrm{s}\right)$ at which the controllers are neither optimized nor tuned.

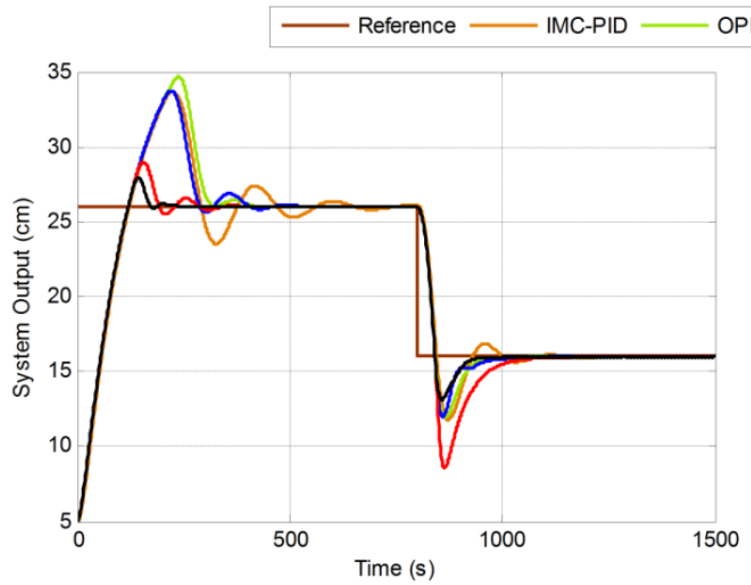

(a)

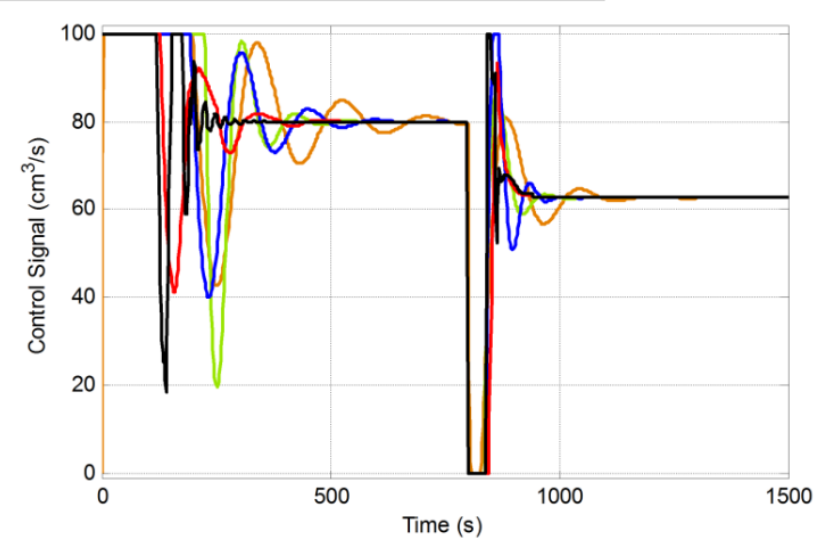

(b)

Fig. 6. Illustration of the (a) process outputs and (b) control signals for the second trajectory control performance. 
At first, a step input disturbance with a magnitude of “ +50 " has been applied the process at $50^{\text {th }}$ second. The control signals and process outputs for the input rejection performances are given in Fig.7. As it can be observed from Fig.7 that, OIT2FPID controller noticeably showed superior performances compared with type-1 and conventional PID counterparts. Secondly, a step output disturbance with a magnitude of "-10" is applied to the process at $1000^{\text {th }}$ second. The control signals and process outputs for the output disturbance rejection performances are given in Fig.8. As it can be seen, the proposed OIT2-FPID control structure compensated very effectively the disturbance in a short period of time compared to the other implemented controller structures. The calculated performance values for the input and output disturbance rejection performances are tabulated in Table V. The performance measures demonstrate that the OIT2-FPID controller is more capable to handle input and output disturbances.

TABLE V. Disturbance Rejection Performance Comparisons

\begin{tabular}{|c|c|c|}
\hline & \multicolumn{2}{|c|}{ IAE } \\
\hline & Input Disturbance & Output Disturbance \\
\hline IMC-PID & 488.5943 & 1039.2654 \\
\hline OPID & 111.4146 & 0697.3698 \\
\hline IMC-T1-FPID & 131.2272 & 0684.8105 \\
\hline OT1-FLCPID & 060.0468 & 0478.0654 \\
\hline OIT2-FLCPID & 004.0262 & 0387.6190 \\
\hline
\end{tabular}

VI. CONCLUISON

In this paper, the $\mathrm{BB}-\mathrm{BC}$ optimization algorithm was used to optimize antecedent membership functions of an interval type2 fuzzy PID controller for a nonlinear cascaded two tank process. The optimization performances of the IT2-FPID controller (20 parameters) was compared with an optimized T1-FPID controller (14 parameters) and an optimized PID controller (3 parameters) based on the IAE performance index. Since the number of optimization parameters of type-2 fuzzy controller is relatively big, we preferred the BB-BC optimization method which has a low computational cost and a high convergence speed. In order to make a fair comparison, the rule base and scaling factors are kept fixed for the fuzzy controller structures while only the antecedent parameters are optimized for a defined reference trajectory. The optimization results demonstrated that the OIT2-FPID, which has more design parameters, outperforms the optimized type-1 fuzzy and conventional PID controller structures with respect to IAE performance index. Since the FOU provides the antecedent type-2 fuzzy sets with extra degrees of freedom, it can be concluded that an IT2-FPID is more capable of providing lower IAE performance measure compared to a type-1 counterpart that has the same rules and scaling factors. In other words, this extra degree of freedom gives the BB-BC global search method an opportunity to find a more optimal solution than those obtained for the optimized OPID and OT1FPID.

The closed loop system performances of the optimized controllers have been also analyzed in detail. In this context, a detailed comparative study is presented on the highly nonlinear cascaded two tank process. The control performance results of the optimized controllers are also compared with IMC-PID and
IMC-T1-FPID controllers based on performance measures which are settling time, overshoot and IAE. At first, it has been illustrated that the OIT2-FPID improves the overall system performance of the process significantly for the reference trajectory at which the controllers are optimized or tuned in every sense. Then, the ability of the controllers to cope with noise, nonlinear dynamics and disturbances is investigated. The performance results of the comparative study show that the OIT2-FPID structure attenuates the disturbances and noises as well as improves transient state of the closed loop response in different operating points even those at which the controller was not optimized.

It can be concluded that the control performance of an IT2FPID can be improved by tuning the FOUs of its antecedent IT2-FSs. Moreover, the optimized IT2-FPID appears to be more robust against nonlinear dynamics, output noise, and input \& output disturbances in comparison with the type-1 fuzzy and conventional PID controller structures. When IT2FPID is tuned offline, this benefit is especially useful in order to reduce the effect of unexpected nonlinear dynamics, noises and disturbances.

Future work will focus on implementing the proposed type2 fuzzy control structure on a real-time process and extending the optimization based design strategy for generalized type- 2 fuzzy logic controller structures.

\section{ACKNOWLEDGMENT}

This research is supported by the project (1059B191200447) of Scientific and Technological Research Council of Turkey (TUBITAK). All of these supports are appreciated.

\section{REFERENCES}

[1] N.N. Karnik, J.M. Mendel and Q. Liang, "Type-2 Fuzzy Logic Systems," IEEE Transaction on Fuzzy Systems, vol. 7 (6), pp. 643-658, 1999.

[2] Q. Liang and J.M. Mendel, "Interval Type-2 Fuzzy Logic Systems: Theory and Design," IEEE Transactions on Fuzzy Systems, vol. 8(5), pp. 535-550, 2000.

[3] T. Kumbasar, I. Eksin, M. Guzelkaya and E. Yesil, "Type-2 fuzzy model based controller design for neutralization processes," ISA Transactions, vol. 51 (2), pp. $277-287,2012$.

[4] H. Hagras, "A Hierarchical Type-2 Fuzzy Logic Control Architecture for Autonomous Mobile Robots," IEEE Transactions on Fuzzy Systems, vol. 12(4), pp. 524-539, 2004.

[5] D. Wu and W. W. Tan, "Genetic Learning and Performance Evaluation of Internal Type-2 Fuzzy Logic Controllers," Engineering Applications of Artificial Intelligence, vol. 19 (8), pp. 829-841, 2006.

[6] R. Martinez, O. Castillo and L.T. Aguilar, "Optimization of Interval Type-2 Fuzzy Logic Controllers for a Perturbed Autonomous Wheeled Mobile Robot Using Genetic Algorithms," Information Sciences; vol. 179 (13), pp. 2158-2174, 2009.

[7] O. Castillo, G. Huesca and F. Valdez, "Evolutionary Computing for Optimizing Type-2 Fuzzy Systems in Intelligent Control of Non-Linear Dynamic Plants," In Proceeding of North American Fuzzy Information Processing Society, pp. 247-251, 2005.

[8] M. Galluzzo, B. Cosenza and A. Matharu, "Control of a Nonlinear Continuous Bioreactor with Bifurcation by a Type-2 Fuzzy Logic Controller," Computers \& Chemical Engineering, vol. 32 (12), pp. 29862993, 2008.

[9] T. Kumbasar, I. Eksin, M. Guzelkaya and E. Yesil, "Interval type-2 fuzzy inverse controller design in nonlinear IMC structure," Engineering Applications of Artificial Intelligence, vol. 24 (6), pp. 996 - 1005, 2011. 
[10] D. Wu and W. W. Tan, "Interval type-2 fuzzy PI controllers: Why they are more robust," IEEE International Conference on Granular Computing, Silicon Valley, August 2010, pp. $802-807,2010$.

[11] M. Aliasghary, I. Eksin, M. Guzelkaya and T. Kumbasar, "Design of an interval type-2 fuzzy logic controller based on conventional PI controller," In: Proc. Int. Conf. MED, pp.627-632, 2012.

[12] D. Wu, "On the Fundamental Differences between Type-1 and Interval Type-2 Fuzzy Logic Controllers," Transactions on Fuzzy Systems vol. 20 (5), pp. $832-848,2012$.

[13] D. Wu, "Twelve Considerations in Choosing between Gaussian and Trapezoidal Membership Functions in Interval Type-2 Fuzzy Logic Controllers," IEEE World Congress on Computational Intelligence, Brisbane, Australia, June 2012, pp. 1 - 8, 2012.

[14] M. B. Biglarbegian, W. W. Melek and J. M. Mendel, "A practical approach for design of PD and PI like Interval Type-2 TSK fuzzy controllers," Proc. Int. Conf. SMC, pp. 255-261, 2009.

[15] O. Castillo and P. Melin, "A review on the design and optimization of interval type-2 fuzzy controllers," Applied Soft Computing, vol. 12 (4), pp. 1267-1278, 2012.

[16] O.K. Erol and I. Eksin, "A New Optimization Method: Big Bang Big Crunch," Advances in Engineering Software, vol. 37 (2), pp.106-111, 2006.

[17] T. Kumbasar, I. Eksin, M. Guzelkaya and E. Yesil, E., “Adaptive Fuzzy Model Based Inverse Controller Design Using BB-BC Optimization Algorithm," Expert Systems with Applications, vol. 38 (10), pp. 1235612364, 2011.
[18] S. Iplikci, "A support vector machine based control application to the experimental three-tank system," ISA Transactions, vol. 49 (3), pp. 376386, 2010.

[19] C.V. Camp, "Design of space trusses using big bang big crunch optimization," Journal of Structural Engineering, vol. 133 (7), pp. 999 1008, 2007.

[20] A. Kaveh, A. and S. Talatahari, "Size Optimization of Space Trusses Using Big Bang-Big Crunch Algorithm," Computers and Structures, vol. 8 (17), pp. 1129-1140, 2009.

[21] D. Wang and A. Kazuo, "Research on Fuzzy I-PD Preview Control for Nonlinear System," JSME International Journal Series C, Special Issue on Advances in Motion and Vibration Control Technology, vol. 46 (3), pp. 1042-1050, 2003.

[22] M. Morari, and E. Zafiriou, "Robust process control,". Englewood Cliffs, NJ: Prentice-Hall, 1989.

[23] S. Skogestad, "Simple analytic rules for model reduction and PID controller tuning," Journal of Process Control, vol. 13(4), pp. 291-309, 2003.

[24] W. Z. Qiao and M. Mizumoto, "PID type fuzzy controller and parameters adaptive method," Fuzzy Sets and Systems, vol. 78 (1), pp. $23-35,1996$

[25] X.-G. Duan, H.-X. Li and H. Deng, "Effective Tuning Method for Fuzzy PID with Internal Model Control," Ind. Eng. Chem. Res., vol. 47 (21), pp. 8317-8323, 2008 .

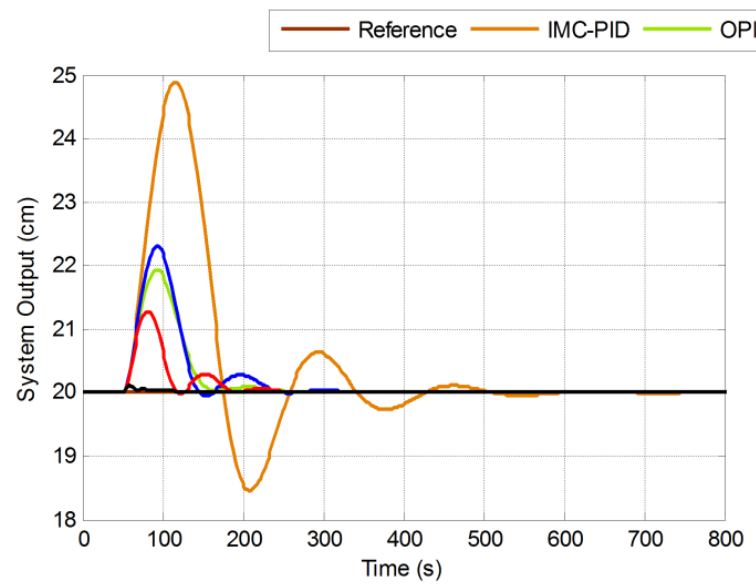

(a)

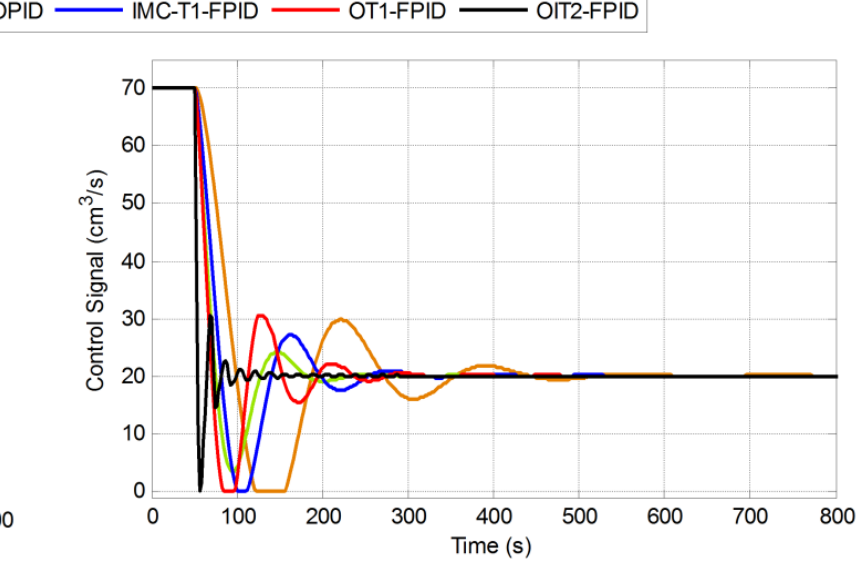

(b)

Fig. 7. Illustration of the (a) process outputs and (b) control signals for the input disturbance rejecetion performance

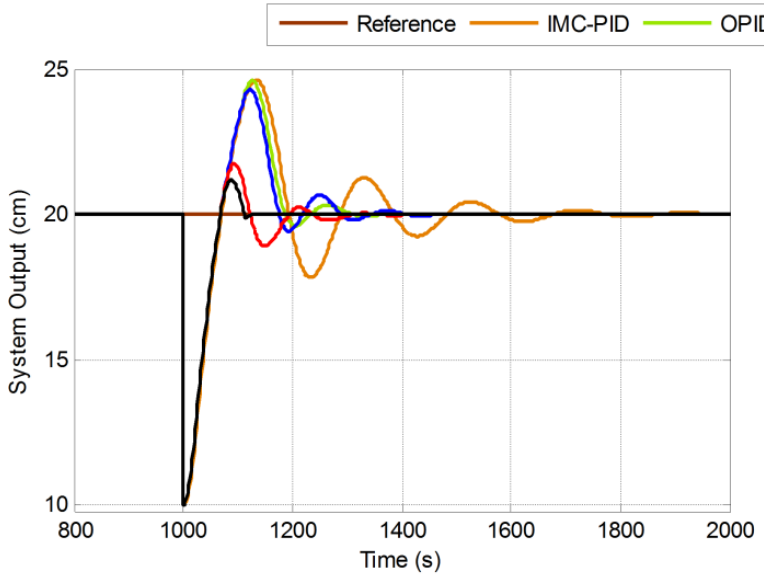

(a)
IMC-T1-FPID — OT1-FPID — OIT2-FPID

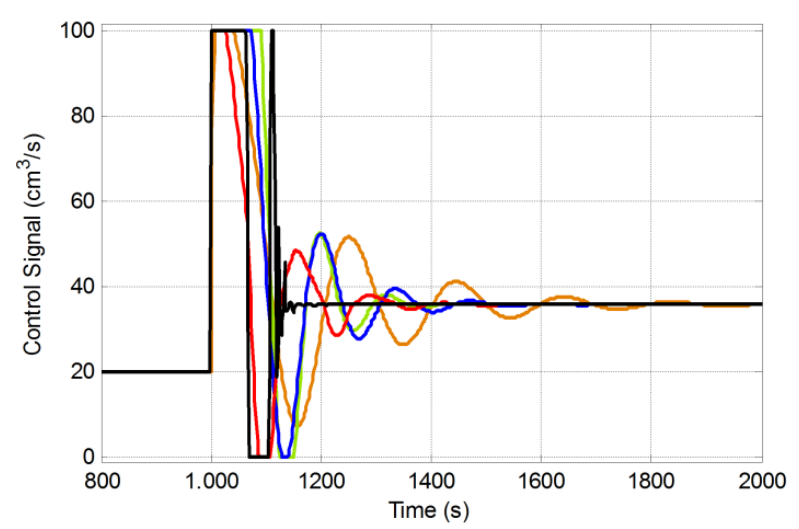

(b)

Fig. 8. Illustration of the (a) process outputs and (b) control signals for the output disturbance rejecetion performance 